ORIGINAL ARTICLE

\title{
A comparative study of continuous positive airway pressure (CPAP) and intermittent positive pressure ventilation (IPPV) in patients with flail chest
}

\author{
M Gunduz, H Unlugenc, M Ozalevli, K Inanoglu, H Akman
}

Emerg Med J 2005;22:325-329. doi: 10.1136/emj.2004.019786

See end of article for authors' affiliations .....................

Correspondence to: Dr M Gunduz, Çukurova University Faculty of Medicine, Department of Anaesthesiology, 01330 , Balcali, Adana, Turkey; hmurat@cu.edu.tr

Accepted 14 November 2004
Introduction: The role of non-invasive positive pressure ventilation delivered through a face mask in patients with flail chest is uncertain. We conducted a prospective, randomised study of continuous positive airway pressure (CPAP) given via a face mask to spontaneously breathing patients compared with intermittent positive pressure ventilation (IPPV) with endotracheal intubation (ETI) in 52 patients with flail chest who required mechanical ventilation.

Method: The 52 mechanically ventilated patients were randomly divided into two treatment groups: the ET group $(n=27)$ received mechanical ventilation with ETI, whereas patients in the CPAP group $(n=25)$ received CPAP via a face mask with patient controlled analgesia (PCA). Major complications, arterial blood gas levels, length of intensive care unit (ICU) stay and ICU survival rate were recorded.

Results: Nosocomial infection was diagnosed in 10 of 21 patients in the ET group, but only in 4 of 22 in the CPAP group $(p=0.001)$. Mean $\mathrm{PO}_{2}$ was significantly higher in the ET group in the first 2 days $(p<0.05)$. There were no significant differences in length of ICU stay between groups. Twenty CPAP patients survived, but only 14 of 21 intubated patients who received IPPV $(p<0.01)$.

Conclusion: Non-invasive CPAP with PCA led to lower mortality and a lower nosocomial infection rate, but similar oxygenation and length of ICU stay. The study supports the application of CPAP at least as a first line of treatment for flail chest caused by blunt thoracic trauma.
$\mathrm{P}$ atients with flail chest often require critical care for analgesia and ventilation. Mechanical ventilation has been used for more than 40 years, ${ }^{1}$ but prolonged ventilation increases the incidence of complications such as ventilator induced lung injury, major atelectasis, and hospital aquired pneumonia. The latter has a poor prognosis, especially in patients with flail chest injury..$^{2-4}$

Trinkle et al proposed the use of epidural analgesia and adequate oxygenation; ${ }^{5}$ however, non-invasive positive pressure ventilation has recently been proposed. For patients with chest wall trauma who remain hypoxic despite adequate regional analgesia and high flow oxygen, continuous positive airway pressure (CPAP) may improve oxygenation and avoid endotracheal intubation. ${ }^{\circ}$

Pain from rib fracture may affect pulmonary function, morbidity, and length of intensive care unit stay. Therefore, effective pain management is a crucial part of therapy for rib fractures by allowing adequate inspiration and clearance of secretions. Previous trials have varied from epidural techniques to patient controlled analgesia (PCA) techniques and have used several outcome variables. However, no significant difference in the lengths of intensive care unit or hospital stays or the incidence of pulmonary complications or organ failure has been reported between epidural and PCA techniques. ${ }^{78}$

In this prospective, randomised study we measured the complications, mortality, oxygenation, and length of intensive care unit (ICU) stay of spontaneously breathing patients with flail chest who required mechanical ventilation given CPAP via a face mask compared with patients given intermittent positive pressure ventilation (IPPV) with endotracheal intubation (ETI).

\section{MATERIALS AND METHODS}

The ethics committee of the Faculty of Medicine of Çukurova University approved the study protocol, and each patient or their next of kin gave informed consent. In total, 52 patients, between the ages of 23 and 49 years, were admitted to our hospital with thoracic trauma between 1 January 2000, and 1 April 2004. Computed lung tomography (CLT) and plain chest $x$ rays were taken routinely on admission, to evaluate patients with multiple traumas. Patients included in the study demonstrated: (a) five or more rib fractures in a row, or three or more segmental (two fractures in one rib) rib fractures on plain chest $x$ ray or CLT and confirmed by the presence of a flail segment-that is, paradoxical motion of the chest wall; (b) acute respiratory distress and severe dyspnoea with respiratory rate $(\mathrm{RR})>25 / \mathrm{min}$; (c) peripheral oxygen saturation $\left(\mathrm{SpO}_{2}\right)<90 \%$ ) while breathing $10 \mathrm{l} / \mathrm{min}$ oxygen in the emergency room; and (c) a ratio of the partial pressure of arterial oxygen to the fraction of inspired oxygen $\left(\mathrm{PaO}_{2} / \mathrm{FiO}_{2}\right) \leqslant 300$ while receiving $\mathrm{FiO}_{2} \geqslant 0.5$ in the ICU.

As mortality is associated with the degree and extent of accompanying injuries, ${ }^{9}$ all patients with flail chest were also assessed using the 25 point Thoracic Trauma Severity Score (TTSS) during the first 2 hours of admission (table 1). ${ }^{10}$ Patients were excluded from the study on the following grounds: requiring ETI immediately on admission due to severe respiratory distress, haemodynamic instability, encephalopathy, respiratory failure caused by neurological disease or asthma attack; emergency surgery following admission; noncooperative patients unable to use the face mask; coma or confusion; inability to protect the airway; severe acidosis,

Abbreviations: CLT, computed lung tomography; CPAP, continuous positive airway pressure; ETI, endotracheal intubation; ETMV, endotracheal intubation and mechanical ventilation; ICU, intensive care unit; IPPV, intermittent positive pressure ventilation; NPPV, non-invasive positive pressure ventilation; PCA, patient controlled analgesia; PEEP, positive end expiratory pressure; RR, respiratory rate; TTSS, Thoracic Trauma Severity Score 


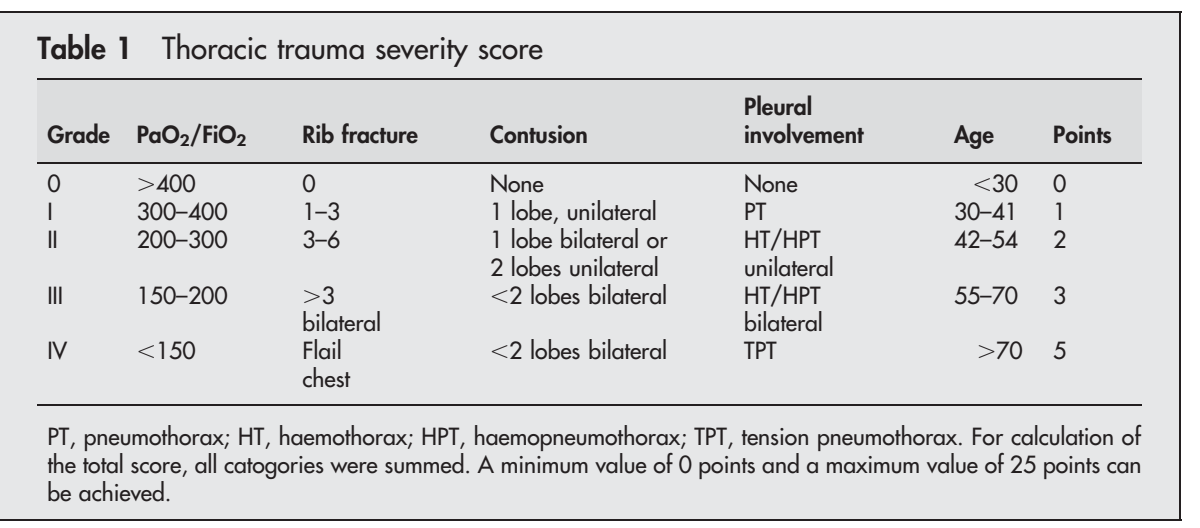

significant co-morbidity; vomiting; obstructed bowel; or haemodynamic instability.

Of the 52 patients, $43(83 \%)$, having a TTSS between 5 and 15 were consecutively randomised to one of two ventilation treatment groups: the ET group $(\mathrm{n}=21)$ received mechanical ventilation with ETI, and the CPAP group $(n=22)$ received CPAP via a face mask. The protocol allowed for CPAP patients to be intubated if symptoms or signs of respiratory distress continued or progressed (respiratory rate $(\mathrm{RR})>25 \mathrm{l} / \mathrm{min}$; $\mathrm{SpO}_{2}<90 \%$ while breathing $10 \mathrm{l} / \mathrm{min}$ oxygen and $\mathrm{PaO}_{2} / \mathrm{FiO}_{2}$ $\leqslant 200$ while receiving $\mathrm{FiO}_{2}=1$.

\section{Mechanical ventilation}

Following ETI, IPPV with positive end expiratory pressure (PEEP) $\left(50-100 \mathrm{mmH}_{2} \mathrm{O}\right)$ was controlled with a tidal volume of $6-10 \mathrm{ml} / \mathrm{kg}, \mathrm{RR} 10 \mathrm{l} / \mathrm{min}$, to produce an end tidal carbon dioxide partial pressure in the range of $4.2-5 \mathrm{kPa}$; hypercapnia was not allowed. Peak inspiratory pressure was limited to $200-300 \mathrm{mmH}_{2} \mathrm{O}$. End expiratory pressure was increased in increments of $20-30 \mathrm{mmH}_{2} \mathrm{O}$ up to $100 \mathrm{mmH}_{2} \mathrm{O}$, until the required $\mathrm{FiO}_{2}$ was $\leqslant 0.6$. Mechanical ventilation was administered with a Drager Evita 4 ventilator.

Following improvement in the clinical condition and blood gas and the ventilatory parameters $\left(\mathrm{RR}, \mathrm{SpO}_{2}\right.$, gas exchange values), when spontaneous breathing reappeared, ventilator settings were changed to intermittent mandatory ventilation (rate 4-7 breaths/min) for weaning from mechanical ventilation. Pressure support ( 140 to $200 \mathrm{mmH}_{2} \mathrm{O}$ ), was adjusted to achieve a spontaneous tidal volume of 8 to $10 \mathrm{ml} / \mathrm{kg} / \mathrm{min}$ and a respiratory rate of $<25$ breaths/min, with accessory muscle activity absent. All patients were weaned from the ventilator by reducing the level of pressure support by $40 \mathrm{mmH}_{2} \mathrm{O}$ twice and then decreasing the ventilatory rate by two breaths/min at 2 hour intervals, as tolerated. Patients who tolerated an intermittent mandatory ventilation rate of 0.5 breaths $/ \mathrm{min}$, a pressure support level of $80 \mathrm{mmH}_{2} \mathrm{O}$, and an $\mathrm{FiO}_{2}$ of $\leqslant 0.5$ then underwent a 2 hour $\mathrm{T}$ piece trial, followed by extubation if respiratory rate remained $<25$ breaths/min and $\mathrm{PaO}_{2}$ $>75 \mathrm{~mm} \mathrm{Hg}$.

As the clinical condition and blood gas and the ventilatory parameters ( $\mathrm{RR}, \mathrm{SpO}_{2}$, gas exchange values) improved, ventilation support was tapered off by progressively reducing the number of daily trials, or was stopped if the improvement remained stable $\left(\mathrm{SpO}_{2}>90 \%, \mathrm{RR}<25\right.$ breaths/min).

\section{Continuous positive pressure ventilation}

Patients in the CPAP group were instructed in the use of the facemask, through which CPAP was delivered by a Drager Evita 4 ventilator. CPAP was increased in increments of 20$30 \mathrm{mmH}_{2} \mathrm{O}$ repeatedly up to $80-150 \mathrm{mmH}_{2} \mathrm{O}$ until the $\mathrm{FlO}_{2}$ requirement was $\leqslant 0.6$. The CPAP level was adjusted on the basis of continuous oximetry and measurements of arterial blood gases.
CPAP was stopped if the respiratory rate was $<25$ breaths/ min, $\mathrm{PaO}_{2}>75 \mathrm{~mm} \mathrm{Hg}$ with a $\mathrm{FiO}_{2}$ of 0.5 , without ventilatory support, and if $\mathrm{PaO}_{2}: \mathrm{FiO}_{2}$ exceeded 200 for 24 hours. Therapy was considered successful if intubation was avoided and the patient could be transferred out of the ICU. Patients received CPAP for at least 6 hours each day, depending on their ability to tolerate it. Each day, however, patients were allowed to breathe spontaneously, with oxygen but without assistance, for 2 hours. The overall duration of CPAP ventilation was determined on the basis of clinical criteria and arterial blood gas levels; in each case, the decision was made by the physician in charge.

\section{Monitoring}

Monitoring of patients in the ICU included lead II electrocardiogram, pulse oximetry, invasive blood pressure, central venous pressure, end tidal $\mathrm{CO}_{2}$ pressure, core temperature, urine output, and ventilator settings (including tidal volume, respiratory rate, lung compliance, airway resistance, peak inspiratory pressure). Ventilator settings were adjusted on the basis of continuous pulse oximetry and measurements of arterial blood gases.

\section{Sedation and analgesia}

In the ET group patients, ETI was facilitated by an intravenous bolus of vecuronium $(0.1-0.2 \mathrm{mg} / \mathrm{kg})$. A propofol $(2-3 \mathrm{mg} / \mathrm{kg} / \mathrm{h})$ plus fentanyl $(0.002 \mu \mathrm{g} / \mathrm{kg} / \mathrm{min})$ combination was infused continuously. Thereafter no neuromuscular blocking agent was used.

In the CPAP group, patients were sedated with an intravenous midazolam infusion $(0.015 \mathrm{mg} / \mathrm{kg} / \mathrm{h})$ and received morphine sulphate patient controlled analgesia. When the patient was breathing spontaneously on admission, and stable vital signs and breathing were maintained under oxygen therapy, a standardised $(0.05 \mathrm{mg} / \mathrm{kg})$ loading dose of morphine was given. Patients were then allowed to use bolus doses of morphine $(0.0125 \mathrm{mg} / \mathrm{kg}$ every 30 minutes without time limit) with a PCA device (Abbott Pain Management Provider, class II, type CF; Abbott, Chicago, IL, USA). Verbal rating scores were measured at 6 hour intervals for 7 days and recorded daily as means (SD), and morphine consumption was recorded daily for 7 days in the CPAP group. Additionally, ondansetron $4 \mathrm{mg}$ intravenously was prescribed every 12 hours on request as the rescue antiemetic therapy. In all patients, chest physiotherapy (percussion and vibration) was initiated following pain control.

\section{Measurements}

All patients with flail chest were assessed on admission using the APACHE III scoring system, and daily thereafter. TTSS and blood count were obtained at the start of the study and chest $x$ rays daily. ${ }^{10}$ Patients were monitored for the development of infections or other complications. Those 
developing manifestations of pneumonia, including radiographic evidence of persistent pulmonary infiltrates, hyperthermia or hypothermia, purulent tracheobronchial secretions, a high white cell count, and worsening of pulmonary gas exchange, underwent bronchoscopy with bronchoalveolar lavage. Pneumonia was diagnosed when at least 100000 colony forming units of bacteria/ml were measured in bronchoalveolar lavage fluids.

TTSS was calculated according to Pape and Remmers. ${ }^{10}$ Arterial blood gas levels were determined at baseline and at $2 \mathrm{~h}$ intervals thereafter for 7 days. Finally, length of ICU stay, ICU survival rate, and the development of major complications associated with IPPV (pneumothorax, nosocomial infections assessed by positive blood culture) or CPAP (pneumothorax, gastric distension) were also recorded.

\section{End points}

In this study, the primary end point was the ICU mortality. Secondary end points included complications associated with IPPV or CPAP as above, oxygenation, and length of ICU stay.

\section{Statistical analysis}

Statistical analyses were performed using the statistical package SPSS (version 10.0; SPSS, Chicago, IL, USA). Results are given as means (SD). Demographic and physiological characteristics of the two groups were compared with Student's $t$ test. Categorical data were analysed by using the $\chi^{2}$ test. Statistical significance was set at $\mathrm{p}<0.05$.

\section{RESULTS}

Of the original 52 patients, nine were excluded from the study: seven required emergency endotracheal intubation because of severe respiratory distress ( $R R>25$ breaths/min, $\mathrm{SpO}_{2}<90 \%$ while breathing $10 \mathrm{l} / \mathrm{min}$ oxygen, and $\mathrm{PaO}_{2} / \mathrm{FiO}_{2}$ $\leqslant 200$ while receiving an $\mathrm{FiO} 2 \geqslant 1$ ), and deterioration in neurological status (Glasgow Coma Scale score of 8 or less) immediately after admission to the intensive care unit, while the other two patients were haemodynamically unstable. The remaining 43 patients were enrolled $(n=21$ in the ET group and $n=22$ in the CPAP group) in this study.

There were no differences in age, sex, and weight between the two groups. APACHE III scores were similar between two groups on admission. Clinical and physiological characteristics on admission to the ICU are shown in table 2 . In the first two days, $\mathrm{PO}_{2}$ was significantly greater in the ET group $(p<0.05)$, but this reduced after 2 days to similar levels to the CPAP group (fig 1). There were no differences in $\mathrm{PCO}_{2}$ between the two groups.

The sedated but conscious CPAP patients experienced some difficulties in adapting to delivery via the face mask for the first 2 days, but thereafter tolerated the treatment well. No patient in this group needed any change in ventilator setting or required intubation. The mean (SD) CPAP level was 100 (20) $\mathrm{mmH}_{2} \mathrm{O}$, and duration of CPAP application was 15 (4) days. Verbal rating scores and morphine consumptions decreased with time in the CPAP group (table 3).

Mean ICU stay was slightly but not significantly longer in the ET group. Mean hospital stay did not differ significantly between groups ( 16 (3) $v 15$ (4) days). Nosocomial infection developed in 10 patients in the ET group: nosocomial pneumonia (streptococcal pneumonia $\mathrm{n}=2$, Staphylococcus aureus $\mathrm{n}=5$, and Klebsiella pneumonia $\mathrm{n}=3$ ), and in four patients in the CPAP group: nosocomial pneumonia (streptococcal pneumonia $\mathrm{n}=2$ ), urinary infection (Escherichia coli $n=2$ ). Seven patients in the ET group and two patients in the CPAP group died from nosocomial infection and related complications. Complications related with death are summarised in table 4. No other complications associated with IPPV (barotrauma) or CPAP (pneumothorax, gastric

\begin{tabular}{|c|c|c|}
\hline Variable & $\begin{array}{l}\text { ET group } \\
(\mathrm{n}=21)\end{array}$ & $\begin{array}{l}\text { CPAP group } \\
(n=22)\end{array}$ \\
\hline $\operatorname{Sex}(F / M)$ & $7 / 14$ & $9 / 13$ \\
\hline Age (years) & $38(10)$ & $40(9)$ \\
\hline APACHE III & $30(5)$ & $32(7)$ \\
\hline Weight (kg) & $71(5)$ & 70 (3) \\
\hline $\mathrm{pO}_{2}(\mathrm{mmHg})$ & $78(15)$ & $106(40)$ \\
\hline $\mathrm{pCO}_{2}(\mathrm{mmHg})$ & $39(3)$ & $32(6)$ \\
\hline $\mathrm{paO}_{2} / \mathrm{FiO}_{2}$ & $200(50)$ & $190(40)$ \\
\hline Length of ICU stay (day) & $16(3)$ & $15(4)$ \\
\hline \multicolumn{3}{|l|}{ Chest wall injuries (n)(\%) } \\
\hline $2-3$ ribs & $8(38 \%)$ & $7(31.8 \%)$ \\
\hline$>3$ ribs & $13(62 \%)$ & $15(68.2 \%)$ \\
\hline HT & $4(19 \%)$ & $6(27.3 \%)$ \\
\hline PT & $9(42.8 \%)$ & $11(50 \%)$ \\
\hline HPT & $3(14.2 \%)$ & $2(9.1 \%)$ \\
\hline TPT & $1(4.76 \%)$ & $0(\%)$ \\
\hline
\end{tabular}

HT, Haemothorax; PT, pneumothorax; HPT, haemopneumothorax; TPT, tension pneumothorax. Data are shown as mean (SD).

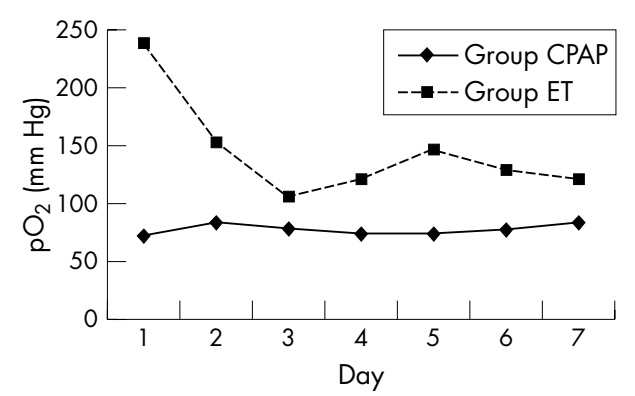

Figure 1 The $\mathrm{PO}_{2}$ in both groups during treatment. In the first two days, $\mathrm{PO}_{2}$ was significantly greater in the ET group (diamonds) $(\mathrm{p}<0.05)$, but this reduced after 2 days, to similar levels to the CPAP group (squares). There were no differences in $\mathrm{PCO}_{2}$ between the two groups.

Table 3 Verbal rating score and morphine consumption in the CPAP group

\begin{tabular}{llllll}
\hline & \multicolumn{3}{l}{ VRS } & & \multicolumn{2}{l}{ Morphine consumption (mg) } \\
\cline { 2 - 3 } \cline { 5 - 6 } Day & Mean (SD) & $\begin{array}{l}\text { Median } \\
\text { (min-max) }\end{array}$ & Mean (SD) & $\begin{array}{l}\text { Median } \\
\text { (min-max) }\end{array}$ \\
\hline 1 & $6.0(0.53)$ & $6(5-7)$ & & $35.40(1.40)$ & $36(30-36)$ \\
2 & $5.36(0.072)$ & $5(4-7)$ & & $31.86(2.62)$ & $32(24-36)$ \\
3 & $4.72(0.88)$ & $4.5(4-7)$ & & $27.72(3.02)$ & $28(20-30)$ \\
4 & $3.95(0.65)$ & $4(3-5)$ & & $19.72(4.37)$ & $18.5(14-28)$ \\
5 & $3.18(0.50)$ & $3(2-4)$ & & $15.81(3.38)$ & $15.5(12-26)$ \\
6 & $2.68(0.56)$ & $3(2-4)$ & $13.77(2.15)$ & $13.5(12-20)$ \\
7 & $2.13(0.46)$ & $2(2-4)$ & $12.40(0.95)$ & $12(12-16)$ \\
\hline
\end{tabular}

distension) were recorded. Clearly, patients receiving CPAP had a better survival $(\mathrm{p}<0.01)$.

\section{DISCUSSION}

This is the first study to evaluate use of CPAP for the treatment of flail chest caused by blunt thoracic trauma. Many other therapeutic approaches, including surgical, have been previously reported. In the past, especially in cases of severe lung contusion and prolonged mechanical ventilation, surgery has been recommended for external stabilisation of the chest wall. ${ }^{11-13}$ The first modern approach to flail chest 


\begin{tabular}{|c|c|c|}
\hline Complication & $\begin{array}{l}\text { ET group } \\
(n=21)(n)(\%)\end{array}$ & $\begin{array}{l}\text { CPAP group } \\
(n=22)(n)(\%)\end{array}$ \\
\hline Pneumonia & $10(47.6 \%)$ & $2(9.1 \%)$ \\
\hline Urinary infection & $0(0 \%)$ & $2(9.1 \%)$ \\
\hline Sepsis & $5(23.8 \%)$ & $2(9.1 \%)$ \\
\hline Renal failure & 4 (19.1\%) & $1(4.5 \%)$ \\
\hline MOF & $1(4.8 \%)$ & $0(0 \%)$ \\
\hline ARDS & $1(4.8 \%)$ & $0(0 \%)$ \\
\hline
\end{tabular}

was that of Avery et al, who reported that continued mechanical ventilation was required to achieve the internal stabilisation of the chest wall. ${ }^{14}$ Cullen et al recommended IMV for stabilisation in the treatment of flail chest injury. ${ }^{15}$ Antonelli et al, however, reported that the mechanical ventilation procedure should be more selective and showed that application of the two different ventilatory techniques (invasive with non-invasive ventilatory treatment) in hypoxaemic respiratory failure resulted in similar short term improvements in arterial blood gases, while non-invasive mechanical ventilation was associated with fewer serious complications and a shorter stay in the ICU compared with conventional mechanical ventilation. ${ }^{16}$

The effectiveness of non-invasive positive pressure ventilation (NPPV) in decreasing mortality and endotracheal intubation rates in patients with hypoxaemic respiratory failure has been demonstrated in meta-analyses, ${ }^{17}$ while the data to support the use of NPPV in these patients with hypoxaemic respiratory failure is inconclusive at present. Several prospective randomised trials have demonstrated that non-invasive ventilation reduces both the need for ETI and the complication rate, and also shortens the length of ICU stay, and improves survival in patients with chronic obstructive pulmonary disease. ${ }^{18}{ }^{19}$ A recent study evaluated whether facemask CPAP is physiologically beneficial, and reduces the need for endotracheal intubation and mechanical ventilation (ETMV) in patients with acute lung injury. ${ }^{20}$ Despite an early favourable physiological response to CPAP in terms of comfort and oxygenation, there were no differences in the need for ETMV, in hospital mortality, or length of ICU stay. However, in a retrospective study, Tanaka et al reported that CPAP with pressure support during spontaneous breathing reduced both complications and the duration of mechanical ventilation. ${ }^{21}$ One randomised controlled trial ${ }^{22}$ and two case series ${ }^{23}{ }^{24}$ support the use of CPAP in isolated chest trauma. The trial was performed on 69 patients with more than two rib fractures and hypoxaemia. ${ }^{22}$ CPAP and regional analgesia were compared with immediate intubation followed by IPPV with PEEP. CPAP resulted in fewer treatment days (mean $4.5 \vee 7.3$ ), fewer mean ICU days $(5.3 \vee 9.5)$, and hospital days $(8.4 v 14.6)$. Kasai et $a^{24}$ also showed that mechanical ventilation led to longer intensive care compared to conservative approaches. In our study, there were no differences between durations of ICU stay between groups, but possibly a greater number of patients would have shown a difference.

Tzelepis et al investigated chest wall distortion in patients with flail chest injury using three different ventilator modes: continuous mandatory ventilation, intermittent mandatory ventilation, and CPAP. ${ }^{25}$ Chest wall distortion was less during spontaneous breathing with CPAP than during intermittent mandatory ventilation. CPAP using a high gas flow system of $80-100 \mathrm{l} / \mathrm{min}$ resulted in the least distortion, possibly related to the positive pleural pressure and minimal load of the high gas flow system. This suggests that CPAP provides more effective stabilisation in flail chest injury than any other positive pressure ventilation. These findings might also be true for CPAP delivered via face mask.

Ventilator related pneumonia, sepsis, septic shock, and other complications have an important effect on mortality in patients with chest trauma and developed flail chest. ${ }^{26-28}$ Pneumonia is the commonest infection in patients with flail chest injury, and bronchial hygiene is an important factor in its prevention. Maintenance of bronchial hygiene is difficult in intubated patients. The lower nosocomial infection rate in the CPAP group suggests that spontaneous breathing with CPAP permits better bronchial hygiene. Respiratory physiotherapy is also useful for bronchial hygiene; in our study, it was initiated following pain control. A lack of adequate bronchial hygiene undoubtedly contributes to pulmonary morbidity. ${ }^{21}$

Pneumothorax is another cause of pulmonary morbidity. It might be suggested that IPPV is more likely to result in barotrauma, ${ }^{22}$ but when CPAP is used in patients with rib fractures, the risk of pneumothorax is similar. ${ }^{6}$ However, only one report describes a case of pneumothorax with CPAP delivered via mask. ${ }^{27}$ There was no overt barotrauma in the present study.

Bauer et $a^{29}$ reported that mechanical ventilation of more than 48 hours' duration is the most important risk factor for ventilator related pneumonias. Our pneumonia rate in the ET group was $48 \%(n=10)$, but only $9 \%(n=2)$ in the CPAP group $(\mathrm{p}<0.001)$, which is probably related to the lower nosocomial infection rate with CPAP. These findings agree with those of uncontrolled studies using CPAP or IPPV;22 2430 however, in a multiple logistic regression model, failure to improve with mask CPAP was an independent predictor of death. $^{31}$

CPAP was less effective in improving $\mathrm{PO}_{2}$ in the first 2 days than IPPV, possibly because of difficulty in adaptation to the CPAP mask. Thereafter, $\mathrm{PO}_{2}$ levels in the CPAP and IPPV groups were similar. Poor oxygenation in patients receiving CPAP is thought to be related to severe pain, analgesia being an important part of the treatment of flail chest. However, as it was difficult for patients with flail chest injury on admission to assume the lateral position for regional analgesic techniques, PCA is an acceptable alternative.

In conclusion, non-invasive CPAP with PCA led to lower mortality and nosocomial infection rate. Oxygenation and length of ICU stay were similar. The findings suggest that CPAP should be tried as a first step in the treatment of flail chest caused by blunt thoracic trauma. Further studies and larger groups are needed to draw firm conclusions and to establish the importance of this protocol in the treatment of flail chest injury.

\section{Authors' affiliations}

M Gunduz, M Ozalevli, H Unlugenc, K Inanoglu, H Akman, Çukurova University Faculty of Medicine Department of Anaesthesiology Balcali, Adana, Turkey;

Competing interests: none declared

\section{REFERENCES}

1 Rodriguez A. Injuries of chest wall, the lung and the pleura. In: Turney SZ, Rodriguez A, Cowley RA, eds. In: Management of cardiothoracic trauma, edn. Baltimore: Williams and Wilkins, 1990:155-77.

2 Paris F, Terrazona V, Garcia-Zarza A. Controversial aspects of surgical fixation for traumatic flail chest. In: International trends of general thoracic surgery. Surgical management of chest injuries. Saunders, Philadelphia, 1991:306-9.

3 Cross AS, Roupe B. Rol of respiratory assistance device in endemic nasocomial pneumonia. Am J Med 1981;70:681-5.

4 DE Lunches LM, Kilinsky VK. Risk factors for pneumonia and fatality in patients receiving continuous mechanical ventilation. Am Rev Respir Dis 1986; 133:792-6.

5 Trinkle JK, Richardson JD, Frenz JL, et al. Management of flail chest without mechanical ventilation. Ann Thoracic Surg 1975;19:355-63. 
6 Boundain S, Blumenthal S, Cooper B, et al (British Thoracic Society Standards of Care Committee). Non-invasive ventilation in acute respiratory failure. Thorax 2002;57:192-211

7 Wu CL, Jani ND, Perkins FM, et al. Thoracic epidural versus intravenous patient-controlled analgesia for the treatment of rib fracture pain after motor vehicle crash. J Trauma 1999;47:564-7.

8 Moon MR, Luchette FA, Gibson SW. Prospective randomized comparison of epidural versus parenteral opioid analgesia in thoracic trauma. Ann Surg 1999;229:684-91.

9 Ciraulo LL, Elliot D, Mitchell KA, et al. Flail chest as a marker for significant injuries. J Am Coll Surg 1994;178:466-70.

10 Pape HC, Remmes D, Rice J. Appraisal of early evaluation of blunt chest trauma: Development of a standardized scoring system for initial clinical decision making. J Trauma 2000;49:496-504.

11 Thomas AN, Blaisdell FW, Lewis FR Jr, et al. Operative stabilization for flail chest after blunt trauma. J Thorac Cardiovasc Surg 1978;75:793-801.

12 Freedland M, Wilson RF, Bender JS, et al. The management of flail chest injury, factors affecting outcome. J Trauma 1990;30:1460-8.

13 Trunkey DD. Chest wall injuries. In: Trauma management: cervicothoracic trauma, 2nd ed. In: Blaisdell FW, Trunkey DD, eds. New York: Thieme, 1994:355-73.

14 Avery EE, Morch ET, Benson DW. Critically crushed chests:A new method of treatment with continous mechanical hyperventilation to produce alkolotic apnea and internal pneumatic stabilitation. J Thorac Surg 1956;32:291-31 1

15 Cullen P, Modell JH, Dirby RR. Treatment of flail chest.Use of intermittent mandatory ventilation and positive end-expiratory pressure. Arch Surg 1975; 110:1099-103.

16 Antonelli M, Conti G, Rocco M, et al. A comparison of noninvasive positive pressure ventilation and conventional mechanical ventilation in patients with acute respiratory failure. N Engl J Med 1998;339:429-35.

17 Peter JV, Moran JL, Phillips-Hughes J, et al. Noninvasive ventilation in acute respiratory failure-A meta-analysis update. Crit Care Med 2002:30:555-62.

18 Wysocki $M$, Tric L, Wolff MA, et al. Noninvasive pressure support ventilation in patients with acute respiratory failure. Chest 1993;103:907-13.
19 Keenan SP, Kernerman PD, Cook DJ, et al. Effect of noninvasive positive pressure ventilation on mortality in patients admitted with acute respiratory failure: a meta analysis. Crit Care Med 1997;25:1685-92.

20 Golberg P, Reissmann H, Maltais F, et al. Efficacy of noninvasive CPAP in COPD with acute respiratory failure. Eur Respir 1995;8:1894-900.

21 Tanaka H, Tajimi K, Endoh Y, et al. Pneumatic stabilization for flail chest injury: an 11 year study. Surg Today 2001;31:12-17.

22 Bolliger CT, Van Eeden SF. Treatment of multipl rip fractures. Randomized controlled trial comparing ventilatory with nonventilatory management. Chest 1990;97:943-8.

23 Hurst JM, DeHaven CB, Branson RD. Use of CPAP mask as the sole mode of ventilatory support in truma patients with mild to moderate respiratory insufficiency. J Trauma 1985;25:1065-8.

24 Linton DM, Potgieter PD. Conservative management of blunt chest trauma. $S$ Afr Med J 1982;61:917-19.

25 Tzelepis GE, McCool FD, Hoppin FG. Chest wall distortion with flail chest. Am Rev Respir Dis 1989;140:31-7.

26 Kingston DW, Phang PT, Leathley MJ. Increased incidence of nosocomial pneumonia in mechanically ventilated patient with subcilinical aspiration. Am J Surg 1991;161:589-92.

27 Craven DE, Steger KA. Epidemiology of nosocomial pneumonia. New perspectives on an old disease. Chest 1995;108(suppl2):1-16S.

28 Gregg RW, Friedman BC, Williams JF, et al. Continuous positive airway pressure by face mak in Pneumocystis Carini pneumonia. Crit Care Med 1990; 18:21-4.

29 Baver TT, Ferrer R, Angrill J, et al. Ventilator associated pneumonia: incidence, risk factors and microbiology. Semin Resrir Infect 2000; 15:272-9.

30 Confalonieri $M$, Calderini $E$, Terraciano S, et al. Noninvasive ventilation for treating acute respiratory failure in AIDS patients with pnuemocystis carinii pneumonia. Intensive Care Med 2002;28:1233-8.

31 Bedos JP, Dumoulin JL, Gachot B, et al. Pneumocystis carinii pneumonia requring intensive care management:survival and prognostic study in 110 patients with human immunodeficiency virus. Crit Care Med 1999;27:1109-15 